\title{
Using lactate dehydrogenase to predict the severity of respiratory distress in term newborn infants with no perinatal asphyxia
}

\author{
Miran Lee ${ }^{1 \oplus}$, Narae Lee $^{1 \odot}$, Mi Hye Bae ${ }^{2 \odot}$, Young Mi Han ${ }^{1 \odot}$, Kyung Hee Park ${ }^{2 \odot}$, \\ Shin Yun Byun ${ }^{1 \oplus}$, Choongrak Kim ${ }^{3 \odot}$ \\ ${ }^{1}$ Department of Pediatrics, Pusan National University Children's Hospital, Pusan National University School of Medicine, Yangsan, \\ South Korea; ' ${ }^{2}$ eepartment of Pediatrics, Pusan National University Hospital, Pusan National University School of Medicine, Busan, \\ South Korea; ${ }^{3}$ Department of Statistics, Pusan National University, Busan, South Korea.
}

\begin{abstract}
Background. We aimed to establish whether knowledge of lactate dehydrogenase (LDH) levels on day 1, as well as the change in these levels in the first three days, could be of clinical benefit in the diagnosis and/or prediction of severity of respiratory distress syndrome (RDS) and transient tachypnea of the newborn (TTN).

Methods. A retrospective study was conducted on 275 term infants (35 with RDS and 240 with TTN) admitted to the neonatal intensive care unit from January 2014 to June 2019. LDH levels were measured on admission and after three days.

Results. Both RDS and TTN groups had elevated LDH levels during admission. LDH levels were significantly higher in the RDS group than in the TTN group on both days. LDH levels in both groups significantly correlated with both the duration of respiratory support required, as well as the number of hospital days. We used these outcomes as a measure of severity of these conditions.

Conclusions. In patients with respiratory distress, it may not be clinically useful to use LDH levels on day 1 to differentiate between RDS and TTN, despite the statistically significant differences, because of the overlapping values. However, LDH levels on day 1 and day 3 may predict the degree and duration of the required respiratory support for both RDS and TTN groups.
\end{abstract}

Key words: lactate dehydrogenase, transient tachypnea of the newborn, neonate, hyaline membrane disease.

Respiratory distress in term neonates occurs relatively frequently, with the two most common differential diagnoses being transient tachypnea of the newborn (TTN) and respiratory distress syndrome (RDS). Although usually a mild and self-limited disease, TTN may require respiratory support. ${ }^{1}$

Lactate dehydrogenase (LDH) is an intracellular enzyme found in the cytoplasm of nearly all human tissues. Injured cells with loss of cell

\footnotetext{
$\bowtie$ Young Mi Han

skybluehym@gmail.com

Received 2nd September 2020,

accepted 31st December 2020.
}

membrane integrity leak their LDH into the surrounding extracellular spaces. Cellular injury in newborns may be a result of different conditions, not only pre- and peri-partum, but also ongoing injury post-partum. Measurement of plasma LDH is routinely available in most hospitals. LDH has been extensively studied in neonates and has been used as a marker of the severity of various conditions. The suggested neonatal LDH cut-off values to identify "general illness" and the need for neonatal intensive care unit (NICU) support are 600 and 800 IU/L for different sensitivities and specificities. ${ }^{2}$ In asphyxiated newborns, initial LDH levels may be useful in predicting the severity of their illness and the subsequent duration of mechanical ventilation. ${ }^{3}$ 
One study of 54 newborns stated that "LDH is an excellent predictor to differentiate RDS from TTN soon after birth in full-term neonates with respiratory distress". ${ }^{4}$ We wanted to look at LDH levels in a larger group of RDS and TTN patients to assess its diagnostic value, as well as its possible predictive value in terms of the degree and duration of respiratory support requirement.

\section{Material and Methods}

\section{Design}

This was a single-center, retrospective study of term infants admitted for respiratory distress without a history of fetal distress or perinatal asphyxia. A pediatric radiology specialist interpreted the chest $\mathrm{X}$-ray. This study was approved by the Institutional Review Board of our hospital and the requirement for informed consent was waived (IRB No. 05-2019-126).

\section{Study population}

This study included term infants (gestational age $\geq 37$ weeks) who were admitted to a tertiary referral neonatal center for respiratory distress, within 24 hours of birth between January 2014 and June 2019, and diagnosed with either TTN or RDS. Both in-house births and transfers into the center were included. The exclusion criteria were: meconium aspiration syndrome, complex cardiac anomalies diagnosed by echocardiogram, lung anomalies diagnosed by chest X-ray, perinatal asphyxia (defined as resuscitation with more than $10 \mathrm{~min}$ of positive pressure ventilation before stable spontaneous respiration, $\mathrm{pH}<7.10$ or base deficit $\geq 16 \mathrm{mmol} / \mathrm{L}$, or Apgar score $<6$ at $5 \mathrm{~min}$ ), chromosomal anomalies, congenital infection including pneumonia and admission after 24 hours' age.

\section{TTN diagnosis}

TTN was diagnosed based on initial clinical signs (onset of tachypnea [respiratory rate exceeding 60 breaths/min] within 6 hours postnatally, persistence of tachypnea for at least
12 hours) and one of the following radiologic findings: lung hyperinflation, prominent pulmonary vascular markings, flattening of the diaphragm, or fluid in the fissures. ${ }^{5}$

\section{RDS diagnosis}

The diagnostic criteria of RDS included clinical features such as grunting, retractions, nasal flaring, cyanosis, tachypnea, an initial (pre-intubation) need for oxygen or pressure support, and radiographic findings such as a diffuse reticulogranular pattern, groundglass appearance and superimposed air bronchograms. $^{6}$

\section{Respiratory stabilization}

Oxygen supplementation was provided to maintain peripheral oxygen saturation $\left(\mathrm{SpO}_{2}\right)$ above $95 \%$. Additional respiratory support was administered via invasive positive pressure mechanical ventilation or non-invasive support, which included nasal continuous positive airway pressure (nCPAP), humidified high flow nasal cannula (HHFNC), or low flow nasal cannula, based on the degree of respiratory requirement.

Mechanical ventilation for the TTN group was determined by the attending physician based on one or more of the following criteria: sustained signs of respiratory distress such as chest retraction accompanied by grunting or nasal flaring and tachypnea ( $>60$ breaths $/ \mathrm{min}$ ), desaturation of $\mathrm{SpO}_{2}<93 \%, \mathrm{PCO}_{2}>60 \mathrm{mmHg}$, or repeated apnea of 20 seconds or longer accompanied by bradycardia $<60$ beats/min. Surfactant was administered to all of the RDS infants, but to none of the TTN infants given their assumed underlying pathophysiology. Extubation criteria were determined by the attending physician and were similar for both RDS and TTN groups.

\section{Data collection and outcome measurement}

Basic information such as gestational age, birth weight, gender, multiple gestation, caesarean section, 1 and 5-minute Apgar scores, maternal 
age, gestational diabetes status, pregnancyinduced hypertension, duration of invasive respiratory support, duration of overall respiratory support and duration of hospital stay were retrospectively collected from the medical records.

All patients underwent a complete blood count, blood chemistry including LDH, aspartate aminotransferase (AST), alanine aminotransferase (ALT), C-reactive protein (CRP) and arterial blood gas analysis (ABGA) on admission (within 24 hours of birth); LDH and CRP were again measured on day 3 of life.

A Nova Critical Care Xpress and a Nova pHOx Ultra analyzers (Nova Biomedical, Waltham, MA, USA) were used to measure ABGA; the measurements included $\mathrm{pH}, \mathrm{pCO}_{2^{\prime}}, \mathrm{pO}_{2^{\prime}}$ base excess (BE), and $\mathrm{HCO}_{3}-$. A TBA-200FR NEO (Toshiba Medical Systems Corporation, Otawara, Japan) and an AU5800® Chemistry Analyzer (Beckman Coulter, Chaska, USA) were used to measure serum LDH, AST, and ALT levels. The receiver operating characteristic (ROC) curve was calculated to assess the usefulness of LDH in the diagnosis of RDS. Regression analysis was performed to assess the association between LDH levels (on admission and on day 3 after admission) and the total number of days on any respiratory support (invasive, non-invasive pressure support, or supplemental oxygen), and duration of hospital stay.

\section{Statistical analysis}

Statistical analyses were performed using SPSS version 21.0 (IBM Co., Armonk, NY, USA). Continuous data are presented as mean and standard deviation and categorical data are presented as frequencies or percentages. The area under the curve (AUC) was obtained from the ROC curve to assess the predictive value of LDH on admission for diagnosis of RDS. The RDS group was compared with the TTN group using the independent Student's $t$-test for continuous data and the approximate z-test for comparing two population proportions.
Logistic regression analysis was performed on the entire study population using $\mathrm{LDH}$ on admission and on day 3 after admission as dependent variables, and total duration of respiratory support requirement and duration of hospital stay as independent variables. Further, linear regression of the total duration of subsequent respiratory support and duration of hospital stay were done on the day $1 \mathrm{LDH}$ level. Statistical significance was considered as a $p$-value $<0.05$.

\section{Results}

Table I contains the demographic characteristics of the study participants. During the study period, 403 patients were admitted for respiratory symptoms. From those, we excluded 39 cases of meconium aspiration syndrome, 10 cases of complex cardiac anomalies, 17 cases of lung anomalies, 23 cases of perinatal asphyxia, and 29 cases of vertical infection including pneumonia. The 10 cases of tachypnea transferred/admitted 24 hours after birth were also excluded. Finally, 275 patients were eligible for the study, of which 173 patients $(62.9 \%)$ were male; $237(86.2 \%)$ were born elsewhere and subsequently transferred to our center. The mean gestational age and weight at birth were $38.6 \pm 1.2$ weeks and 3270.7 $\pm 473.2 \mathrm{~g}$, respectively. The mean Apgar scores at 1 and 5 minutes were $7.4 \pm 1.4$ and $8.7 \pm 1.1$, respectively; no differences were found between both groups.

We diagnosed 35 patients with RDS and 240 with TTN. The mean gestational age ( $37.9 \pm 0.7$ weeks vs. $38.7 \pm 1.2$ weeks, respectively; $p<0.001$ ) was significantly lower in the RDS group than that of the TTN group. In contrast, the frequency of Caesarean sections (31/35 [88.6\%] vs. 131/240 [54.6\%]; $p<0.001)$ and mean maternal age (34.8 \pm 4.4 years vs. $32.4 \pm 4.6$ years; $\mathrm{p}=0.003$ ) were both significantly higher in the RDS group.

Blood tests on admission revealed that both groups had elevated LDH levels (RDS group: $1337.2 \pm 285.3 \mathrm{IU} / \mathrm{L} ;$ TTN group: $1205.6 \pm 399.0$ 
Table I. Demographic characteristics of the study participants.

\begin{tabular}{|c|c|c|c|c|}
\hline & Total $(n=275)$ & $\operatorname{RDS}(\mathrm{n}=35)$ & TTN (n=240) & $p$-value \\
\hline Gestational age (weeks) & $38.6 \pm 1.2$ & $37.9 \pm 0.7$ & $38.7 \pm 1.2$ & $<0.001$ \\
\hline Birth weight (g) & $3270.7 \pm 473.2$ & $3151.2 \pm 406.6$ & $3288.1 \pm 480.5$ & 0.109 \\
\hline Male (\%) & $173(62.9)$ & $20(57.1)$ & $153(63.7)$ & 0.569 \\
\hline Twin $(\%)$ & $10(3.6)$ & $1(2.9)$ & $9(3.8)$ & 1 \\
\hline Caesarean section (\%) & $162(58.9)$ & $31(88.6)$ & $131(54.6)$ & $<0.001$ \\
\hline Apgar score (1 min) & $7.4 \pm 1.4$ & $7.5 \pm 1.8$ & $7.4 \pm 1.4$ & 0.684 \\
\hline Apgar score (5 min) & $8.7 \pm 1.1$ & $8.7 \pm 1.5$ & $8.7 \pm 0.9$ & 0.983 \\
\hline Maternal age & $32.7 \pm 4.6$ & $34.8 \pm 4.4$ & $32.4 \pm 4.6$ & 0.003 \\
\hline GDM (\%) & $16(5.8)$ & $1(2.9)$ & $15(6.2)$ & 0.678 \\
\hline PIH (\%) & $5(1.8)$ & $0(0)$ & $5(2.1)$ & 0.853 \\
\hline
\end{tabular}

Values are expressed as mean \pm standard deviation or number $(\%)$.

GDM: gestational diabetes mellitus, PIH: pregnancy-induced hypertension, RDS: respiratory distress syndrome, TTN: transient tachypnea of the newborn.

Table II. Comparison of the laboratory test results on admission and on day 3 after admission between the RDS and TTN groups.

\begin{tabular}{lccc}
\hline & RDS $(\mathrm{n}=35)$ & TTN $(\mathrm{n}=240)$ & -value \\
\hline On admission & & & \\
$\mathrm{AST}(\mathrm{IU} / \mathrm{L})$ & $67.0 \pm 21.5$ & $69.9 \pm 42.7$ & 0.524 \\
$\mathrm{ALT}(\mathrm{IU} / \mathrm{L})$ & $11.3 \pm 14.6$ & $12.5 \pm 7.8$ & 0.438 \\
$\mathrm{LDH}(\mathrm{IU} / \mathrm{L})$ & $1337.2 \pm 285.3$ & $1205.6 \pm 399.0$ & 0.019 \\
$\mathrm{CRP}(\mathrm{mg} / \mathrm{dL})$ & $0.05 \pm 0.06$ & $0.31 \pm 0.78$ & $<0.001$ \\
$\mathrm{pH}$ & $7.35 \pm 0.07$ & $7.37 \pm 0.08$ & 0.165 \\
$\mathrm{PCO}_{2}(\mathrm{mmHg})$ & $38.0 \pm 8.8$ & $33.6 \pm 8.5$ & $<0.001$ \\
$\mathrm{PO}_{2}(\mathrm{mmHg})$ & $98.2 \pm 51.8$ & $94.4 \pm 54.8$ & 0.697 \\
$\mathrm{BE}_{\mathrm{HCO}}-(\mathrm{mMol})$ & $-4.86 \pm 1.77$ & $-5.31 \pm 3.16$ & 0.242 \\
$3 \mathrm{rd} \mathrm{day}$ & $20.4 \pm 2.3$ & $19.1 \pm 3.3$ & $<0.001$ \\
$\mathrm{LDH}(\mathrm{IU} / \mathrm{L})$ & & & \\
$\mathrm{CRP}(\mathrm{mg} / \mathrm{dL})$ & $1560.7 \pm 354.6$ & $1234.2 \pm 459.2$ & $<0.001$ \\
\hline
\end{tabular}

Values are expressed as mean \pm standard deviation.

ALT: alanine aminotransferase, AST: aspartate aminotransferase, BE: base excess, CRP: C-reactive protein, LDH: lactate dehydrogenase, RDS: respiratory distress syndrome, TTN: transient tachypnea of the newborn.

Table III. Comparison of the respiratory support requirement and duration of hospital stay between RDS and TTN groups.

\begin{tabular}{lccc}
\hline & RDS (n=35) & TTN (n=240) & p-value \\
\hline Need for oxygen support (\%) & $34(97.1)$ & $222(92.5)$ & 0.512 \\
Total duration of any respiratory support (days) & $13.1 \pm 6.4$ & $5.5 \pm 4.8$ & $<0.001$ \\
Hospital day (days) & $15.6 \pm 7.3$ & $9.2 \pm 5.4$ & $<0.001$ \\
\hline
\end{tabular}

Values are expressed as mean \pm standard deviation or number (\%).

RDS: respiratory distress syndrome, TTN: transient tachypnea of the newborn. 
$\mathrm{IU} / \mathrm{L})$ when compared to reference values of normal LDH of 500-700 IU/L; there was a statistically significant difference between both groups. ${ }^{7,8} \mathrm{C}$-reactive protein (CRP), $\mathrm{pCO}_{2^{\prime}}$ and $\mathrm{HCO}_{3}$ - were significantly different between both groups, but the levels were within normal ranges, therefore, considered irrelevant for this study. On day 3, the LDH levels (Table II) were, again, significantly higher in the RDS group than the TTN group (RDS group: $1560.7 \pm 354.6$ IU/L; TTN group: $1234.2 \pm 459.2$ IU/L).

There was no significant difference in the need for oxygen supplementation between the two groups. However, the RDS group had a significantly greater total duration of any respiratory support (RDS: $13.1 \pm 6.4$ days vs. TTN: $5.5 \pm 4.8$ days, $\mathrm{p}<0.001$ ). All 35 of the RDS infants were intubated for surfactant administration, as opposed to $49 / 240$ infants with TTN requiring intubation as described above in "respiratory stabilization". One RDS infant received INSURE (intubation-surfactantextubation). The mean duration of hospital stay (RDS: $15.6 \pm 7.3$ days vs. TTN: $9.2 \pm 5.4$ days, $\mathrm{p}<0.001)$ was significantly higher in the RDS group than the TTN group (Table III).

The area under the ROC curve of the LDH level on admission was 0.648 (Fig. 1). To assess the usefulness of LDH levels on admission and on day 3 after admission, the correlation with the following two variables was investigated: (1) duration of total respiratory support requirement, and (2) duration of hospital stay. Logistic regression analysis showed the LDH levels, both at admission and after 3 days, were significantly correlated with both variables, LDH levels on day 3 after admission showing a particularly strong correlation (Table IV). The univariate analysis of LDH level on admission and the two variables showed significant correlations (Fig. 2). Finally, to see the predicting ability of the fitted linear regression (i.e., the ability of the level of LDH at day 1 as a predictor of both variables), we used 200 observations as trainsets and 75 as test sets. As shown in Figure 3 , the LDH level was significant in predicting both variables.

\section{Discussion}

LDH levels are highest soon after birth, generally decreasing with age throughout childhood. ${ }^{9}$ This may be related to the general vulnerability of tissues/organs in the newborn, temporary hypoxia during the birth process, and increased cell membrane permeability and

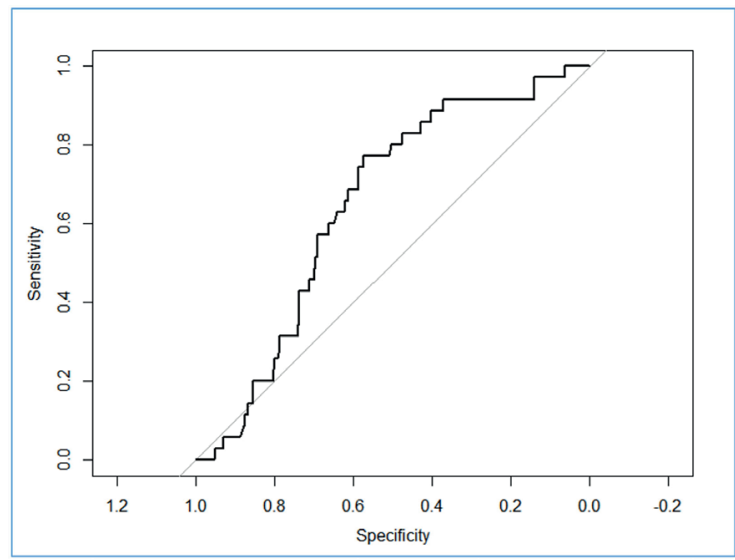

Fig. 1. ROC curve for the value of $\mathrm{LDH}$ as a predictor of RDS. The AUC is 0.648 .

Table IV. Correlations between LDH levels and prognosis (respiratory support requirement and duration of hospital stay) in patients with respiratory distress: logistic regression between LDH and four prognosis variables.

\begin{tabular}{|c|c|c|c|c|}
\hline \multirow[b]{2}{*}{ Response variable } & \multicolumn{2}{|c|}{ On admission } & \multicolumn{2}{|c|}{ Day 3} \\
\hline & $\begin{array}{c}\text { Increase of } \\
\text { response variable } \\
\text { with LDH } 100 \\
\text { increase }\end{array}$ & $p$-value & $\begin{array}{c}\text { Increase of } \\
\text { response variable } \\
\text { with LDH } 100 \\
\text { increase }\end{array}$ & $p$-value \\
\hline Total respiratory support (days) & 0.405 & $<0.001$ & 0.629 & $<0.001$ \\
\hline Hospital stay (days) & 0.345 & $<0.001$ & 0.523 & $<0.001$ \\
\hline
\end{tabular}

LDH: lactate dehydrogenase. 

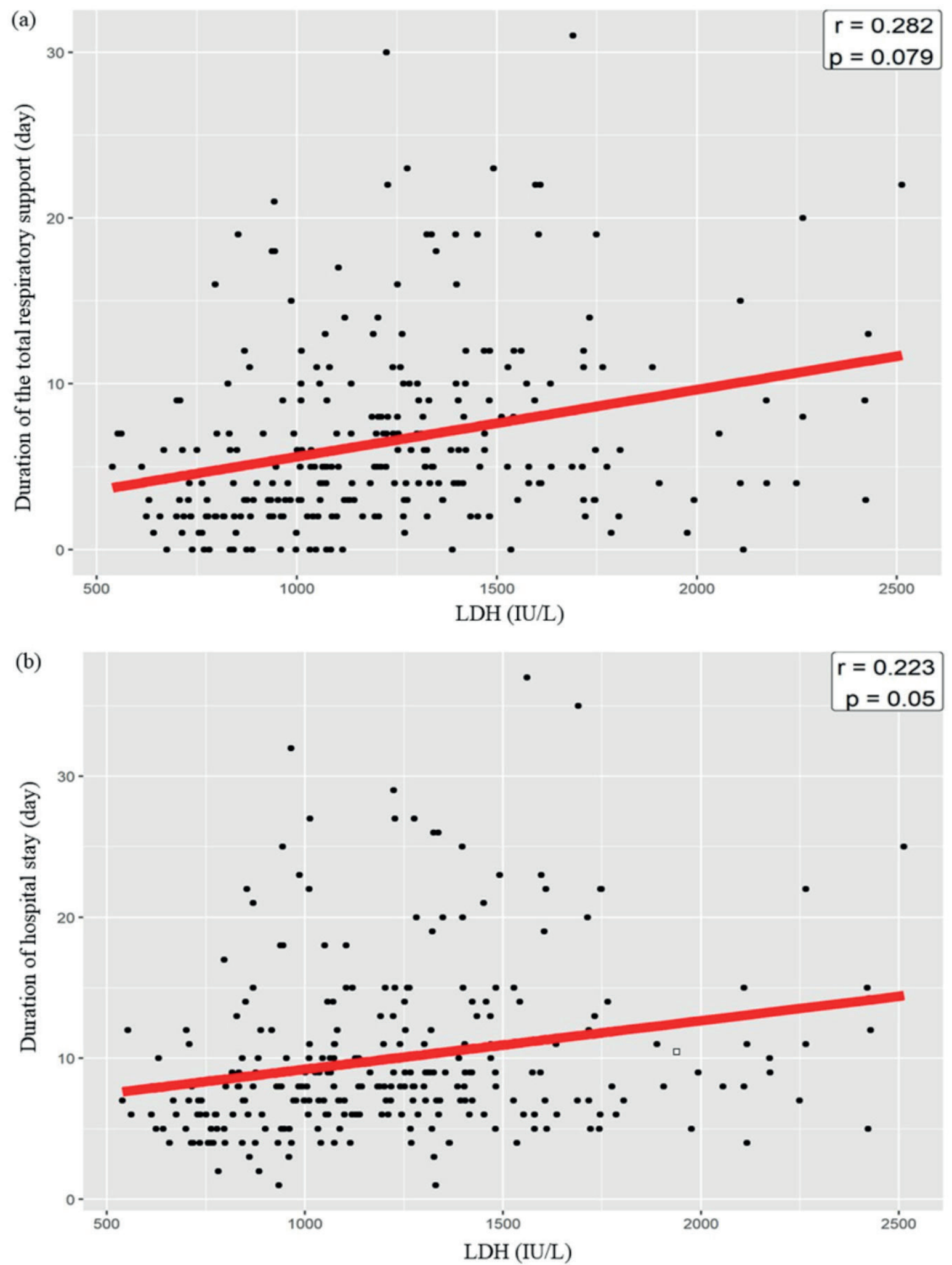

Fig. 2. In patients with respiratory distress, correlation between the LDH level on admission and the total respiratory support requirement (a), and the duration of hospital stay (b). A straight line is the fitted simple linear regression. 

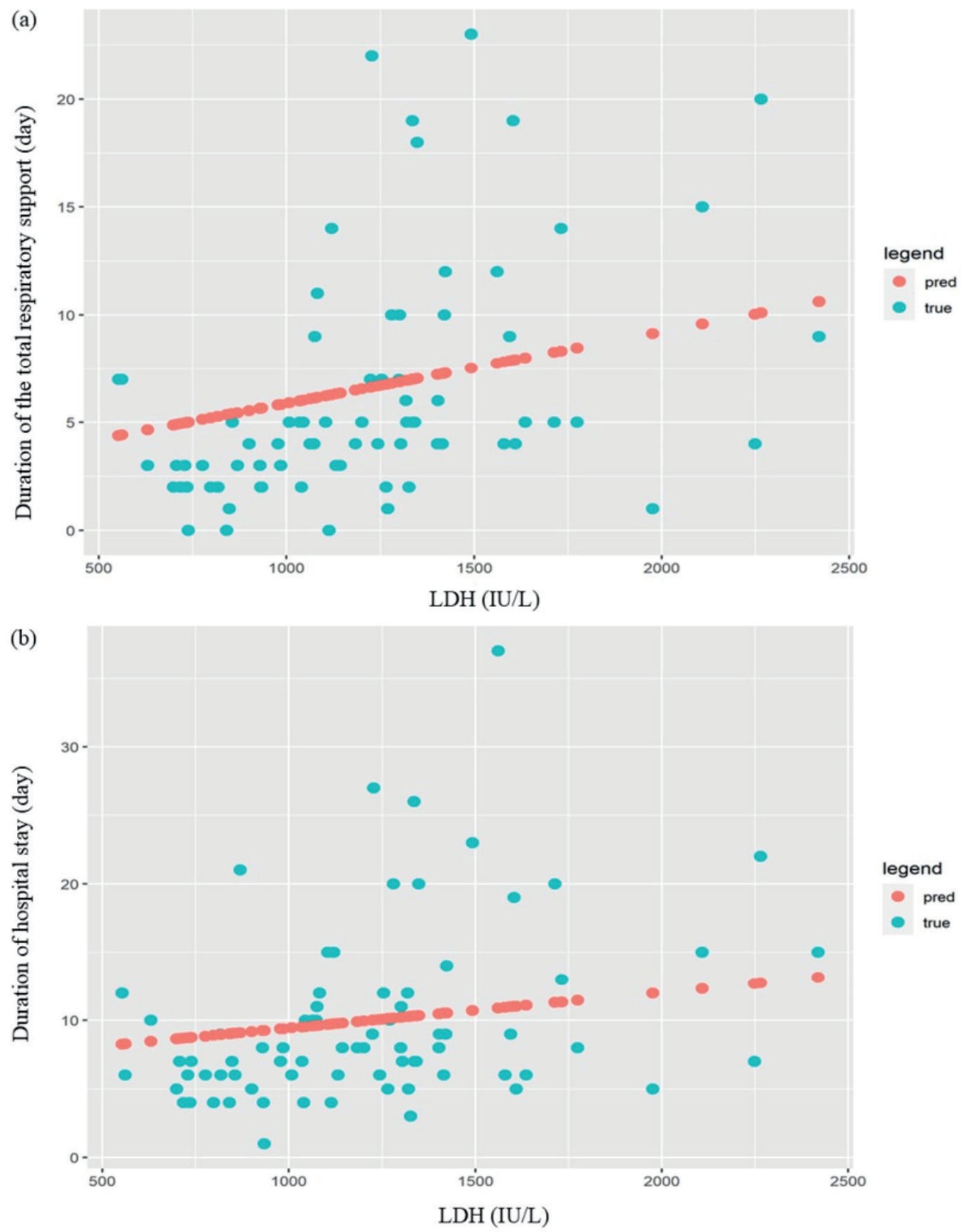

Fig. 3. Using the fitted model based on the 200 trainset, we compare the predicted value (orange) for the 75 test set with true value (green): the total respiratory support requirement (a), and the duration of hospital stay (b). A straight line is the fitted simple linear regression. 
hemolysis after birth. In term healthy newborns, LDH peaks in the first 24 hours, then gradually decreases; with median LDH 358.5 IU/L at birth, $512.0 \mathrm{IU} / \mathrm{L}$ at 24 hours, and $431.0 \mathrm{IU} / \mathrm{L}$ at 72 hours. ${ }^{8}$ For healthy non-asphyxiated newborns, normal LDH levels are between 330-700 IU/ L in the first 24 hours, and between 320-600 IU/L at 72 hours. ${ }^{7,8}$

Because perinatal hypoxia results in plasma LDH elevation postpartum, it is important to identify infants with some degree of perinatal hypoxia to appropriately interpret their postnatal LDH levels. We used the term "perinatal hypoxia" to denote some degree of oxygen deficiency, if insufficient to satisfy the "perinatal asphyxia" criteria defined earlier.

It is not always possible to obtain a reliable history, especially for newborns delivered at another institution. Historically, the Apgar score is an indicator of perinatal hypoxia. A low 5-minute Apgar score alone was correlated to elevated $\mathrm{LDH} .{ }^{10}$ The negative predictive value of the 5-minute Apgar score is the more reliable indicator; wherein a score of 7 or greater, "it is unlikely" that peripartum hypoxia would cause neonatal encephalopathy. ${ }^{11}$ The mean 5-minute Apgar score for our cohort was 8.7.

Arterial $\mathrm{pH}$ and base excess (BE) at or soon after birth have been used as measures of prenatal hypoxia, ${ }^{12}$ until later studies have shown lactate to be a more useful indicator. ${ }^{13-15}$ In our study, cord arterial lactate would have been ideal to identify the presence and severity of perinatal hypoxia, but this is not routinely performed in many institutions, including ours. Postnatal arterial lactate, even within the first 24 hours, may normalize rapidly and thus no longer reflect preceding perinatal events. ${ }^{15}$ Interestingly, there is a strong correlation between lactate and LDH levels on admission in sick neonates. ${ }^{2}$

"Physiological hypoxia" is a spectrum with no current accepted definition. Because perinatal hypoxia can affect the brain and all other body organs, an elevated LDH with some degree of perinatal hypoxia is a non-specific "marker" for hypoxic injury. The most commonly reported injuries secondary to asphyxia are those involving the kidneys and the brain; however, other organ systems, including the lungs, are also directly affected. ${ }^{16}$ Once released from the cells, usually due to stresses of birth, plasma $\mathrm{LDH}$ reduction is dependent on the clearance capacity of the liver and possibly on ongoing cell injury, and thus, continuing LDH release.

Both LDH and AST in normal newborns were inversely related to $\mathrm{pH}$ and $\mathrm{BE}$, which suggested that the more significant the hypoxia, the higher the LDH and AST levels. ${ }^{7}$ Gunes showed increasing elevation of LDH with an increasing degree of perinatal asphyxia. Their mean AST on day 1 was higher in the mild ( $84 \mathrm{U} / \mathrm{L})$, moderate $(160 \mathrm{U} / \mathrm{L})$, and severe asphyxia (227 U/L) groups compared to their control (70 U/L) group. ${ }^{17} \mathrm{We}$ showed a mean AST 67.0 and $69.9 \mathrm{U} / \mathrm{L}$ in our RDS and TTN groups respectively, suggesting that the elevated LDH in our study group was not secondary to significant perinatal hypoxia. In order to further eliminate the possibility of perinatal hypoxia accounting for our raised LDH levels on admission, we performed the same analysis on a subgroup of 138 patients whose AST was within the accepted laboratory reference range $(<60 \mathrm{U} / \mathrm{L})$. The linear regression of the duration of total respiratory support requirement and duration of hospital stay, done on the day $1 \mathrm{LDH}$ level, showed the same results; but an even more statistically significant correlation than what we found from our total patient pool.

Studies on perinatal asphyxia with or without HIE consistently show that the LDH in newborns with moderate and severe asphyxia peaks on day 3 after which it gradually decreases, normalizing within 10 days of life. ${ }^{2,10,17}$ In mildly asphyxiated newborns, the elevated day $1 \mathrm{LDH}$ decreased by day 3. 10,17 Thus, persistently raised and increasing LDH levels on day 3 in both our respiratory distress groups would be explained by perinatal asphyxia only if it was moderate or severe; however, we had no demonstrable perinatal asphyxia. Therefore, our increasing LDH levels would most likely be due to the respiratory conditions themselves: presumably 
secondary to associated lung cellular injury, peripheral organ effects associated with the respiratory conditions, or possibly even by cellular injury caused by the administered respiratory support.

To tease out LDH elevation secondary to primarily respiratory disease, it is notably absent or immeasurable if there is a pre-existing raised LDH secondary to multi-organ hypoxia. Idiopathic respiratory distress syndrome (IRDS) superimposed on perinatal asphyxia does not further alter the already raised LDH and AST levels. ${ }^{18}$ Thus, the cellular lung injury during global asphyxia may already have resulted in an early release of their LDH, which may be greater than any subsequent LDH leak associated with any coexistent respiratory pathology.

Although the first 24-hour LDH has been shown to be a predictor of "general illness" and a need for NICU support, only a few of the admitted IRDS infants had initially elevated LDH values, and IRDS infants were almost always admitted early. ${ }^{2}$ This is important, because Lackmann et al. ${ }^{8}$ normal values show a significant increase in LDH from birth to the 12-hour measurement. In our study, most newborns were born at another institution, so their admission LDH levels, although all within the first 24 hours of age, were not taken soon after birth. Additionally, their respiratory status may not have been ideal prior to admission at our institution. Our initial LDH levels on admission may thus, not reflect the condition of the patients at birth, but rather the condition secondary to the (possibly suboptimally treated) respiratory condition.

Thus, can an elevated LDH be due to respiratory distress, or is it simply a reflection of preceding perinatal hypoxia? Aydogdu et al. ${ }^{19}$ animal model showed that serum enzymes, including $\mathrm{LDH}$, are elevated in cases of respiratory distress syndrome without preceding perinatal hypoxia. Ozkiraz et al. ${ }^{5}$ showed that an initial LDH level, with cut-off value of $750 \mathrm{U} / \mathrm{L}$, offered good predictive value for prolonged oxygen requirement in TTN patients. These authors suggested that initial lactate and LDH values may be useful to identify newborns who may subsequently deteriorate and require respiratory support. Lim's animal model demonstrated a progressive increase of $\mathrm{LDH}$ during ventilator-induced lung injury, showing a negative correlation with oxygenation, suggesting that LDH levels could be an early marker of primary lung injury. ${ }^{20}$

Our term newborns had no measurable perinatal asphyxia, but we cannot exclude some degree of possible mild perinatal hypoxia. LDH levels increasing in the first three days (an expected trend in moderate/severe perinatal asphyxia) in our cohort must clearly be due to postnatal direct lung cellular injury, or to generalized hypoxic effects (including changes in circulation) secondary to the respiratory disease process.

The weaknesses of our study are inherent to its retrospective nature. As standard protocol in most institutions did not include routine measurement of cord arterial lactate levels, we could not definitively assess perinatal hypoxia using this parameter. Cord arterial lactate would be an ideal parameter to include in future studies.

Our study suggests that a raised LDH on day 1 may reflect respiratory distress in the absence of perinatal asphyxia. The day $1 \mathrm{LDH}$ levels were $1337.2 \pm 285.3$ for RDS and 1205.6 \pm 399.0 for TTN, suggesting a range of 1051.91622.5 and 806.6-1604.6, respectively. Since there is considerable overlap in these ranges, LDH levels alone are not clinically useful to differentiate RDS from TTN on day 1, even if there was a statistically significant difference between these groups. However, LDH levels on day 1 in both groups of patients with respiratory distress may predict the degree and duration of the required respiratory support and hospital stay. The significantly higher day $3 \mathrm{LDH}$ in RDS compared to TTN may be predictive of a longer expected duration of respiratory support and hospital stay in the RDS group. The higher LDH in the RDS group may also reflect the degree of ongoing injury on day 3 , as these infants usually take a few days to improve. 


\section{Author contribution}

The authors confirm contribution to the paper as follows: study conception and design: Young Mi Han, Choongrak Kim; data collection: Miran Lee, Narae Lee, Mi Hye Bae; analysis and interpretation of results: Miran Lee, Young Mi Han, Kyung Hee Park, Shin Yun Byun, Choongrak Kim; draft manuscript preparation: Miran Lee, Young Mi Han, Choongrak Kim. All authors reviewed the results and approved the final version of the manuscript.

\section{Ethical approval}

This study was approved by the Institutional Review Board of our hospital and the requirement for informed consent was waived (IRB No. 05-2019-126).

\section{Source of funding}

There is no specific funding.

\section{Conflict of interest}

The authors declare no conflict of interest.

\section{REFERENCES}

1. Edwards MO, Kotecha SJ, Kotecha S. Respiratory distress of the term newborn infant. Paediatr Respir Rev 2013; 14: 29-36.

2. Karlsson M, Dung KT, Thi TL, et al. Lactate dehydrogenase as an indicator of severe illness in neonatal intensive care patients: a longitudinal cohort study. Acta Paediatr 2012; 101: 1225-1231.

3. Nakajima J, Tsutsumi N, Nara S, et al. Correlations of enzyme levels at birth in stressed neonates with short-term outcomes. Fetal Pediatr Pathol 2018; 37: 157-165.

4. An YS, Kim IU, Yang MY, Jeong HR, Kim HS. Serum enzymes in predicting transient tachypnea of newborn and respiratory distress syndrome. Korean J Perinatol 2014; 25: 284-291.
5. Ozkiraz S, Gokmen Z, Boke SB, et al. Lactate and lactate dehydrogenase in predicting the severity of transient tachypnea of the newborn. J Matern Fetal Neonatal Med 2013; 26: 1245-1248.

6. Agrawal V, David RJ, Harris VJ. Classification of acute respiratory disorders of all newborns in a tertiary care center. J Natl Med Assoc 2003; 95: 585595.

7. Mongelli M, Kwan Y, Kay LL, Hjelm M, Rogers MS. Effect of labour and delivery on plasma hepatic enzymes in the newborn. J Obstet Gynaecol Res 2000; 26: 61-63.

8. Lackmann GM. Reference values for selected enzyme activities in serum from healthy human neonates. Clin Biochem 1996; 29: 599-602.

9. Loh TP, Metz MP. Trends and physiology of common serum biochemistries in children aged 0-18 years. Pathology 2015; 47: 452-461.

10. Sharma D, Choudhary M, Lamba M, Shastri S. Correlation of Apgar score with asphyxial hepatic injury and mortality in newborns: a prospective observational study from India. Clin Med Insights Pediatr 2016; 10: 27-34.

11. American Academy of Pediatrics Committee on Fetus and Newborn, American College of, Obstetricians and Gynecologists Committee on Obstetric Practice. The Apgar Score. Pediatrics 2015; 136: 819-822.

12. Ross MG, Gala R. Use of umbilical artery base excess: algorithm for the timing of hypoxic injury. Am J Obstet Gynecol 2002; 187: 1-9.

13. Gjerris AC, Staer-Jensen J, Jorgensen JS, Bergholt T, Nickelsen C. Umbilical cord blood lactate: a valuable tool in the assessment of fetal metabolic acidosis. Eur J Obstet Gynecol Reprod Biol 2008; 139: 16-20.

14. Deshpande SA, Platt MP. Association between blood lactate and acid-base status and mortality in ventilated babies. Arch Dis Child Fetal Neonatal Ed 1997; 76: F15-F20.

15. Shah S, Tracy M, Smyth J. Postnatal lactate as an early predictor of short-term outcome after intrapartum asphyxia. J Perinatol 2004; 24: 16-20.

16. Perlman JM, Tack ED, Martin T, Shackelford G, Amon E. Acute systemic organ injury in term infants after asphyxia. Am J Dis Child 1989; 143: 617-620.

17. Gunes T, Ozturk MA, Koklu SM, Narin N, Köklü E. Troponin-T levels in perinatally asphyxiated infants during the first 15 days of life. Acta Paediatr 2005; 94: 1638-1643. 
18. Lackmann GM. Influence of neonatal idiopathic respiratory distress syndrome on serum enzyme activities in premature healthy and asphyxiated newborns. Am J Perinatol 1996; 13: 329-334.

19. Aydogdu U, Yildiz R, Guzelbektes H, Coskun A, Sen I. Cardiac biomarkers in premature calves with respiratory distress syndrome. Acta Vet Hung 2016; 64: $38-46$
20. Lim SC, Kim YI. The role of the lactate dehydrogenase and the effect of prone position during ventilatorinduced lung injury. J Korean Med Sci 2004; 19: 223228. 